\title{
Curved Hexagonal Packings of Equal Disks in a Circle
}

\author{
B. D. Lubachevsky and R. L. Graham \\ Mathematical Sciences Research Center, AT\&T Bell Laboratories, \\ 600 Mountain Avenue, Murray Hill, NJ 07974, USA \\ bdl@bell-labs.com \\ rlg@research.att.com
}

\begin{abstract}
For each $k \geq 1$ and corresponding hexagonal number $h(k)=3 k(k+1)+1$, we introduce $m(k)=\max \{(k-1) ! / 2,1\}$ packings of $h(k)$ equal disks inside a circle which we call the curved hexagonal packings. The curved hexagonal packing of 7 disks $(k=1$, $m(1)=1)$ is well known and one of the 19 disks $(k=2, m(2)=1)$ has been previously conjectured to be optimal. New curved hexagonal packings of 37, 61, and 91 disks $(k=3$, 4 , and $5, m(3)=1, m(4)=3$, and $m(5)=12$ ) were the densest we obtained on a computer using a so-called "billiards" simulation algorithm. A curved hexagonal packing pattern is invariant under a $60^{\circ}$ rotation. For $k \rightarrow \infty$, the density (covering fraction) of curved hexagonal packings tends to $\pi^{2} / 12$. The limit is smaller than the density of the known optimum disk packing in the infinite plane. We found disk configurations that are denser than curved hexagonal packings for 127, 169, and 217 disks ( $k=6,7$, and 8).

In addition to new packings for $h(k)$ disks, we present the new packings we found for $h(k)+1$ and $h(k)-1$ disks for $k$ up to 5, i.e., for 36, 38, 60, 62, 90, and 92 disks. The additional packings show the "tightness" of the curved hexagonal pattern for $k \leq 5$ : deleting a disk does not change the optimum packing and its quality significantly, but adding a disk causes a substantial rearrangement in the optimum packing and substantially decreases the quality.
\end{abstract}

\section{Introduction}

Patterns of dense geometrical packings are sensitive to the geometry of the enclosing region of space. In particular, dense packings of equal nonoverlapping disks in a circle are different from those in a regular hexagon, as one might expect (see, e.g., [1], [4], or [11]). In this paper, for each $k \geq 1$ and the corresponding hexagonal number $h(k)=$ $3 k(k+1)+1$, we present a pattern of packings of $h(k)$ equal disks in a circle which can be viewed as a "curved" analogue of the densest packing of $h(k)$ disks in a regular hexagon (see Fig. 1.1). For a particular $k$, there exists a set of $m(k)=\max \{(k-1) ! / 2,1\}$ different curved hexagonal packings of the same quality. A curved hexagonal packing 


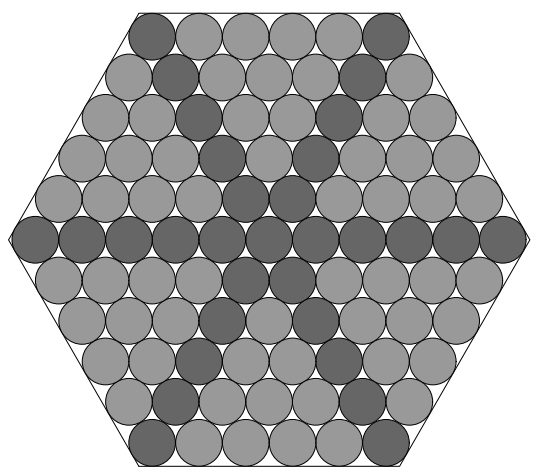

91 disks

density $=0.88434871149353$

$D / d=11.154700538379$

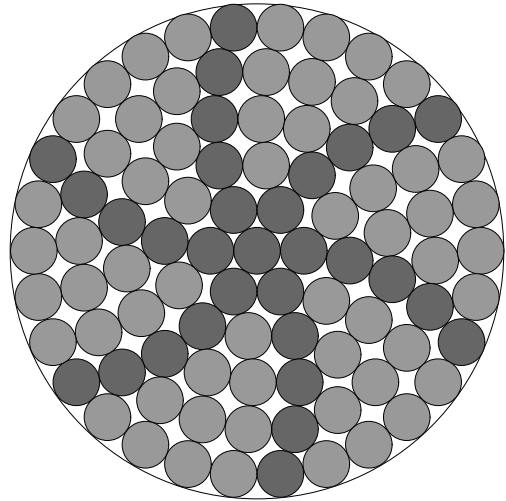

91 disks density $=0.81499829406214$ $D / d=10.566772233506$

Fig. 1.1. Left. The well-known best packing of $h(5)=91$ disks in a regular hexagon. Right. One of the twelve best (that we found) packings of $h(5)=91$ disks in a circle. In both diagrams, the density shown is the ratio of the area covered by disks to the area of the container, and $D$ and $d$ are the diameters of the container and of the disks, respectively. (The different shading of the disks is auxiliary; it is not a part of the pattern.)

pattern is invariant under a $60^{\circ}$ rotation. The density (covering fraction) of a curved hexagonal packing tends to $\pi^{2} / 12$ as $k \rightarrow \infty$. Because this limit is smaller than the density of the best (hexagonal) packing of equal disks on an infinite plane, the curved hexagonal packing of $h(k)$ disks cannot be optimal for sufficiently large $k$.

It is remarkable, though, that for several initial values of $k$ there seems to be no better packing than the curved hexagonal ones. Indeed, for 7 disks $(k=1, m(1)=1)$ the curved hexagonal packing is well known to be optimal and the one for 19 disks $(k=2$, $m(2)=1$ ) has been previously conjectured as such [7]. For 37, 61, and 91 disks $(k=3$, 4 , and 5, $m(3)=1, m(4)=3$, and $m(5)=12$ ), the curved hexagonal packings were the densest we obtained by computer experiments using the so-called "billiards" simulation algorithm.

The "billiards" simulation algorithm [8], [9] has so far proved to be a reliable method for generating optimal packings of disks in an equilateral triangle [5]. Our experiments with this algorithm for packings in a circle either confirmed or improved the best previously reported packings for $n \leq 25$. We are unaware of any published conjectures for packing $n>25$ disks in a circle, but the "billiards" algorithm kept producing packings for many $n>25$, specifically, for $n=h(3)=37, n=h(4)=61$, and $n=h(5)=91$. (A detailed account of these experiments merged with the experiments of Nurmela and Östergård will be reported in a forthcoming paper [6].) The latter three sets of packings happened to have the curved hexagonal pattern and they were the best found for their value of $n$. As for $n=h(6)=127, n=h(7)=169$, and $n=h(8)=217$, the algorithm found packings which are better than the corresponding curved hexagonal packings. 
For the values $k \leq 5$, for which the densest packings of $h(k)$ disks in a circle apparently have the curved hexagonal patterns, these packings look "tight." To test our intuition of their "tightness" we compared these packings with packings obtained for $h(k)-1$ and $h(k)+1$ disks. Thus, we generated dense packings for 36, 38, 60, 62, 90, and 92 disks and verified that deleting a disk does not change the optimum packing and its quality significantly, but adding a disk causes a substantial rearrangement in the optimum packing and substantially decreases the quality. This tightness may be considered an analogue of the similar tightness property for the infinite classes of packings in an equilateral triangle as noted in [5]. Specifically, the variations in the packing pattern and quality when one disk is added or subtracted are similar to those observed for packings of $k(k+1) / 2$ disks in the triangles.

\section{Packings of $7,19,37$, and 61 Disks in a Circle}

The four best packings are presented in Fig. 2.1. The packing of 7 disks is well known and is easily seen to be optimal ${ }^{1}$ and that of 19 disks is conjectured as such in [7]. The packings shown of 37 and 61 disks have not been reported before; they are the best we found for these numbers of disks. The density 7/9 of the 7-disk packing is presented in decimal form in conformance with the other three densities; an alternative finite form of the parameters for all the packings in Fig. 2.1 exists and is discussed in Section 3.

\section{The Curved Hexagonal Pattern}

The pattern can be explained by comparing it with the corresponding hexagonal pattern. Figure 1.1 depicts the two patterns side-by-side for $h(5)=91$ disks. Each is composed of six sections which we tried to emphasize by shading more heavily the disks on the boundaries between them. In the true hexagonal packing, the sections are triangular, and the boundaries are six straight "paths" that lead from the central disk to the six extreme disks. In the curved hexagonal packing, the triangular sections are "curved" and so are the paths.

To define the entire structure of the curved hexagonal packing, it suffices to define the positions of disks on one path. Let the central disk be labeled 0 and the following disks on the path be successively labeled $1,2, \ldots, k$. Consider $k$ straight segments connecting the centers of the adjacent labeled disks: 0 to 1,1 to $2, \ldots,(k-1)$ to $k$. Given a direction of rotation (it is clockwise in Fig. 1.1), each following segment is rotated at the same angle, let us call it $\alpha$, in this direction with respect to the previous segment. Consider $\alpha$ as a parameter. When $\alpha=0$ we have the original hexagonal packing at the left. If we gradually increase the $\alpha$, "humps" grow on the six sides of the hexagon. When $\alpha$ reaches

\footnotetext{
${ }^{1}$ Indeed, if we partition the circle (of radius $r$ ), which contains the centers of the disks, into six equal sectors, then one sector must contain at least two disk centers. The distance between the two centers is at most $r$, and the maximum is achieved when either one disk is centered exactly at the enclosing circle center or when two disks are centered at the peripheral corners of the sector. If needed, we rotate the partition to exclude the latter possibility. Then the central disk position defines the rest of the packing pattern.
} 


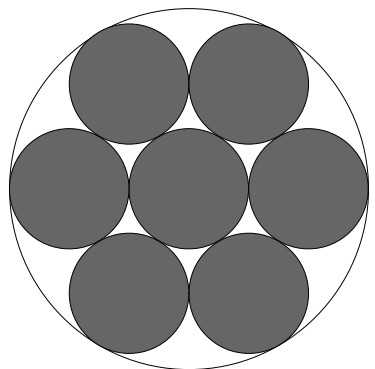

7 disks

density $=0.77777777777778$

$D / d=3.00000000000000$

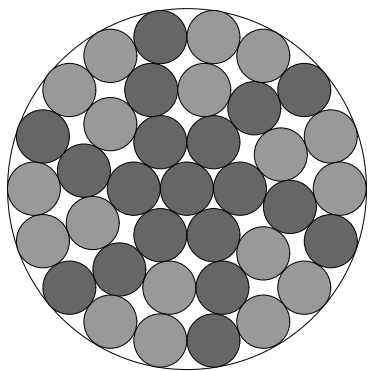

37 disks

density $=0.80996513779820$

$D / d=6.7587704831436$

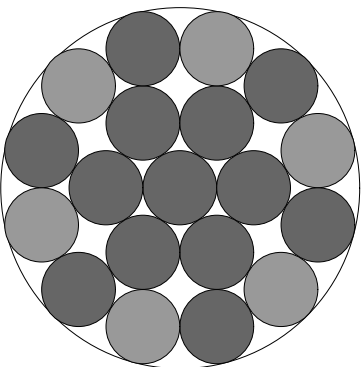

19 disks

density $=0.80319214461341$

$D / d=4.8637033051563$

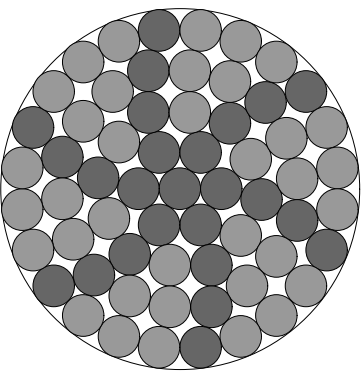

61 disks

density $=0.81313735972642$

$D / d=8.6612975755404$

Fig. 2.1. Top. The well-known best packing of $h(1)=7$ disks and the previously conjectured best packing of $h(2)=19$ disks. Bottom. The best (that we found) packing of $h(3)=37$ disks and the best (one of the three that we found) packing of $h(4)=61$ disks.

the value $\alpha_{k}=\pi / 3 k$, all the disks of the last, $k$ th, layer are at the same distance from the central disk.

Following the path of labeled disks as defined above, the distance $P$, in disk diameters ( $d$ ) from the center of disk 0 to the center of disk $k$, is given by

$$
P=\left|1+e^{i \alpha_{k}}+e^{2 i \alpha_{k}}+\cdots+e^{(k-1) i \alpha_{k}}\right| .
$$

Since $\alpha_{k}=\pi / 3 k$, this simplifies to

$$
P=\frac{1}{2 \sin (\pi / 6 k)}
$$

It follows that the ratio $D / d$ of the enclosing circle diameter to the disk diameter for the curved hexagonal packing is

$$
\frac{D}{d}=1+\frac{1}{\sin (\pi / 6 k)}
$$


The packing density, i.e., the fraction of the enclosing circle area which is covered by disks, can then be found as

$$
\text { density }=h(k)(d / D)^{2} \text {. }
$$

The density tends to the limit $\pi^{2} / 12=0.822467033 \ldots$ as $k \rightarrow \infty$.

The limit density of the curved hexagonal packing is exactly the square of $\pi / 2 \sqrt{3}=$ $0.906899682 \ldots$, the density of the optimum hexagonal packing of the infinite plane (see [2], [3], [5], or [10]). The fact that the latter density is larger than the former implies that the curved hexagonal pattern is nonoptimal for large $k$. This is so, because as $n$ increases, the density of the hexagonal configurations of $n$ congruent disks that fit inside a circle, arbitrarily closely approximates $\pi / 2 \sqrt{3}$ (see [2]). Indeed, we already found better packings for $k=6,7$, and 8 which we discuss in Section 5 .

A curved hexagonal packing of $h(k)$ disks can be constructed for any $k \geq 1$. Figure 3.1 depicts an instance for $k=13$. We believe there are a total of $m(k)=\max \{(k-1) ! / 2,1\}$ noncongruent equally good curved hexagonal patterns of $h(k)$ disks. A curved hexagonal packing pattern is invariant under a $60^{\circ}$ rotation. A method to generate different curved hexagonal packings of a $k$-layered pattern, that is, for $h(k)$ disks, is as follows. Take the pattern described above-let us call it a basic pattern for brevity-and choose a subset among layers $2,3, \ldots, k-1$. Flip the sense of rotation of the chosen layers (or of the corresponding segments on the labeled path). The flip is equivalent to making the mirror reflection of these layers. The flipped layers will fit in their place because of the mirror symmetry of segments of the layers enclosed between consecutive paths in the basic pattern. Note that layers 1 and $k$ are not subject to the flip, because the disks are positioned on them invariantly for all curved hexagonal packings for the given $k$. The resulting $2^{k-2}$ combinations yield $2^{k-3}$ different packings ignoring the reflection
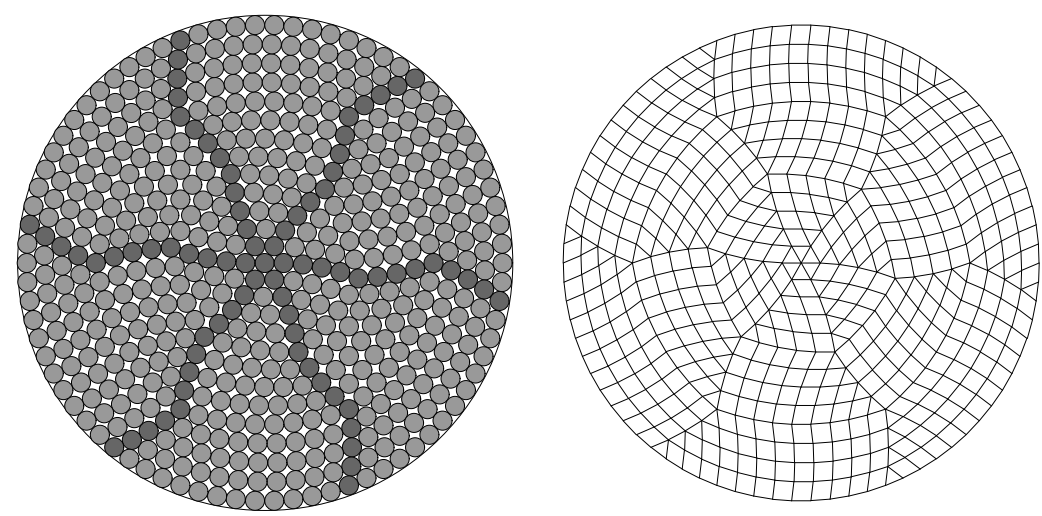

547 disks (13 layers)

density $=0.81954773488724 \quad D / d=25.834885197829$

Fig. 3.1. Left. A curved hexagonal packing of $h(13)=547$ disks in a circle. In this packing the sense of rotation is flipped for layers $6,7,8$, and 9 . Right. The bond diagram for the packing at the left. The diagram contains a straight segment between centers of any pair of disks that are in contact. 
symmetry. We call these modifications "regular" curved hexagonal packings; Fig. 3.1 displays one of them.

We separate regular packings into a distinctive class because their existence follows from the existence of the basic regular packing and is easy to understand. Besides, the regular packings are the most frequent ones among the optimal packings spontaneously generated by our "billiards" procedure (see Section 4). However, the regular packings do not exhaust all the variants since $2^{k-3}<m(k)$ for $k \geq 4$. We believe that the packings in the full set can be identified by different permutations in the order of summands $e^{i \alpha_{k}}, e^{2 i \alpha_{k}}, \ldots, e^{(k-1) i \alpha_{k}}$ in the expression (1) for $P$, or simply by permutations in the sequence

$$
1,2, \ldots, k-1 \text {. }
$$

For each permuted sequence, a path of $k+1$ disks can be constructed and this path, when completed with layers, happens to form a curved hexagonal packing which is equal in quality to the basic one. Permutation $i_{1}, \ldots, i_{k-1}$ of sequence (5) produces the mirror reflection to the pattern produced by permutation $k-i_{1}, \ldots, k-i_{k-1}$.

The method described above fills the pattern layer-by-layer beginning from the central disk out. An alternative method ${ }^{2}$ fills the layers in the opposite direction. The outermost layer contains $6 k$ disks densely placed at the periphery. (Examining the outermost layer is an alternative way to infer (2).) Once layer $k$ is in place, we choose a spot to attach the first disk of layer $k-1$ so that the disk contacts two disks of layer $k$. There are $6 k$ different attachment spots. We attach second, third, . ., 6(k-1)th disk, say, clockwise, so that each next disk contacts the previous disk and at least one disk of layer $k$. As a result exactly 6 disks of layer $k-1$, namely, disk 1 , disk $k$, disk $2 k-1$, disk $3 k-2$, disk $4 k-3$, and disk $5 k-4$ will each contact two disks of layer $k$, while the remaining $6(k-2)$ disks will each contact only one disk of layer $k$. All two-layer configurations thus obtained are congruent to each other by rotation. However, beginning with layer $k-2$ (when $k \geq 3$ ), as we fill the pattern inward, the choice of the spot for the placement of the "first" disk (the one that contacts two disks of the previous layer) distinguishes the pattern, modulo a $60^{\circ}$ rotation. Thus, we have $k-1$ different ways to place the first disk in layer $k-2$, then $k-2$ different ways to place the first disk in layer $k-3$, and so on. This process yields $(k-1)$ ! different clockwise placements. Congruence by the reflection symmetry causes us to half the total: we have $(k-1) ! / 2$ noncongruent placements (for $k \geq 3$ ).

One can enumerate curved hexagonal packings, either by the sequence of rotation angles on a path beginning from the central disk outward, or by the sequence of relative positions of "first" disks beginning from the external layer inward. For either method a simple computer program can be written that synthesizes a curved hexagonal packing given the sequence as an input. In what follows, we adopt the former enumeration because the program we wrote uses that method. Note that we do not offer here a formal proof that either construction method described above actually works, i.e., produces $(k-1)$ !/2 different packings (for $k \geq 3$ ), as claimed, but we strongly believe it does.

The basic packing of 61 disks depicted in Fig. 2.1 corresponds to the original sequence $1,2,3$ or its reflection $3,2,1$. The packings of 61 disks that correspond to permuted

\footnotetext{
${ }^{2}$ Suggested by the anonymous referee.
} 


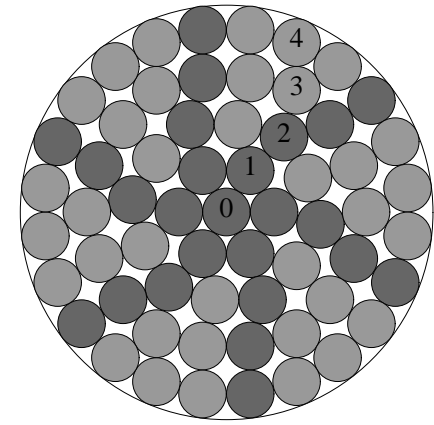

61 disks; permutations $1,3,2$ and 3,1,2

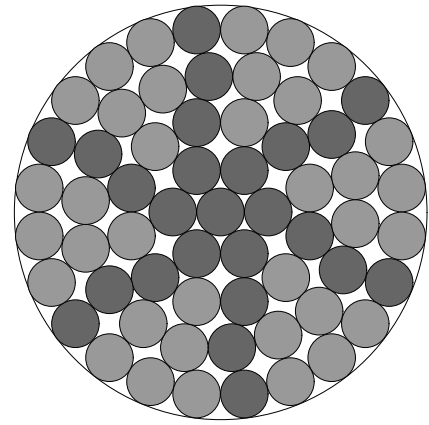

61 disks; permulations 2, 3, 1 and 2, 1,3

Fig. 3.2. Two out of the three existing packings of 61 disks in a circle; the third packing which corresponds to permutations 1, 2, 3 and 3, 2, 1 is given in Fig. 2.1. The role of permutations is explained in the text. The packing at the left is regular which is demonstrated by the path of disks labeled $0,1,2,3$, and 4, each of which has a triangular hole attached. The packing at the right is irregular.

sequences 1,3,2 and 2,3,1 or their reflections 3,1,2 and 2,1,3, respectively, are shown in Fig. 3.2. A characteristic feature of a regular packing is the existence of a path, any disk on which has a triangular hole attached. On the bond diagram, which has a straight segment between the centers of any pair of disks that are in contact (see an example in Fig. 3.1), this path is seen as a (broken) line of $k$ segments that leads from the center to the periphery, each segment on which is a side of a triangle. We identified such a path in the packing at the left in Fig. 3.2 by labeling its disk components. Hence this packing is regular. The one at the right is irregular, because no required path can be found for it. The basic packing of 61 disks shown in Fig. 2.1 is also regular. This amounts to three different packings of $h(4)=61$ disks, two packings of which are regular. Indeed, $m(4)=3$ and $2=2^{4-3}$. Our belief is that for $n=61$ there is no better packing than the three presented. We also believe that there is no fourth packing equal in quality to the three presented but distinct from any of them.

Similarly, there are $m(5)=12$ curved hexagonal packings of $h(5)=91$ disks, $4=$ $2^{5-3}$ of which are regular. One regular packing is represented in Fig. 1.1; it corresponds to the original sequence $1,2,3,4$ or its reflection $4,3,2,1$. The three other regular packings correspond to permuted sequences $(1,2,4,3),(1,4,2,3)$, and $(1,4,3,2)$ or their reflections (resp. $(4,3,1,2),(4,1,3,2)$, and $(4,1,2,3))$. While the existence of the additional regular packings is obvious and those packings are not shown, for a reader who might be not fully convinced in the formal validity of the construction procedures described above we show eight irregular packings in Figs. 3.3 and 3.4. Each packing is accompanied by its two generating sequences and its bond diagram. The bond diagrams clearly distinguish the packings and also show that the packings are irregular. Again, we believe there is no packing better than those 12 and there is no thirteenth packing equal in quality to those 12 but distinct from any one of them.

For $k>5$ there are packings of $h(k)$ disks that are better than the $m(k)$ curved hexagonal ones. It is therefore easy to produce, for such $k$, infinitely many different 

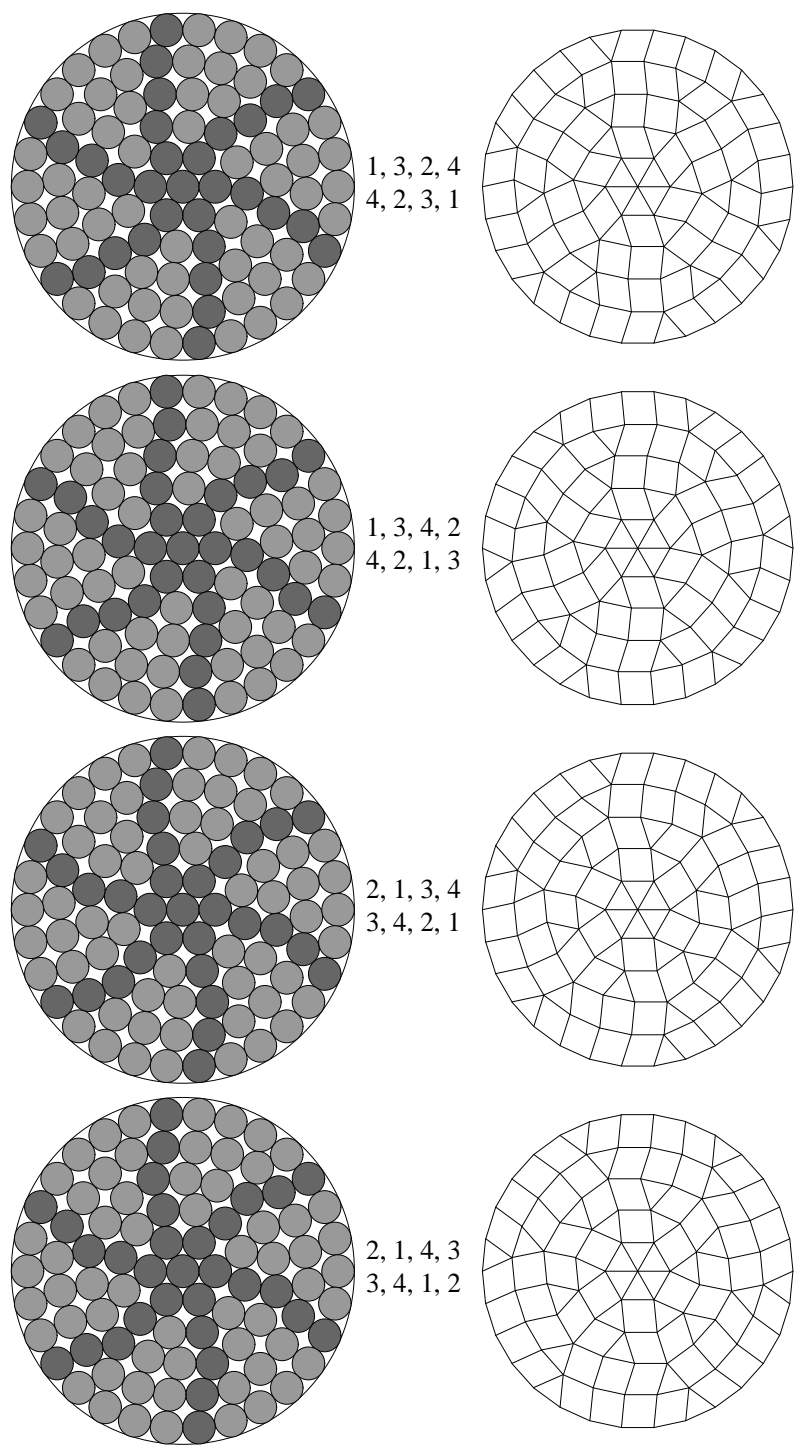

Fig. 3.3. First four irregular densest (that we found) packings of 91 disks. Each packing shown on the left is accompanied by its two generating sequences in the middle and the bond diagram on the right.

configurations of nonoverlapping disks that are equal in quality to curved hexagonal ones. We believe, however, that if a configuration of $h(k)$ congruent disks of the same quality as a curved hexagonal packing of the same disks has a general structure of a curved hexagonal packing described below, then it must be one of the $m(k)$ curved hexagonal packings. The general structure consists of $k$ layers that surround the central disk; layer $i$ consists of $6 i$ disks placed in a circular fashion, for $i=1,2, \ldots, k$, a disk 


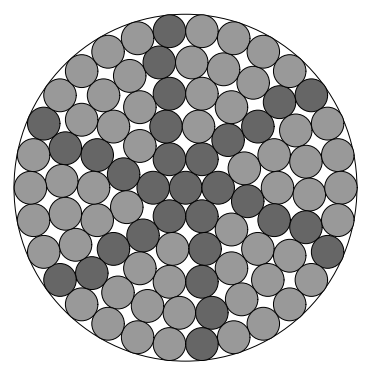

$2,3,1,4$

$3,2,4,1$
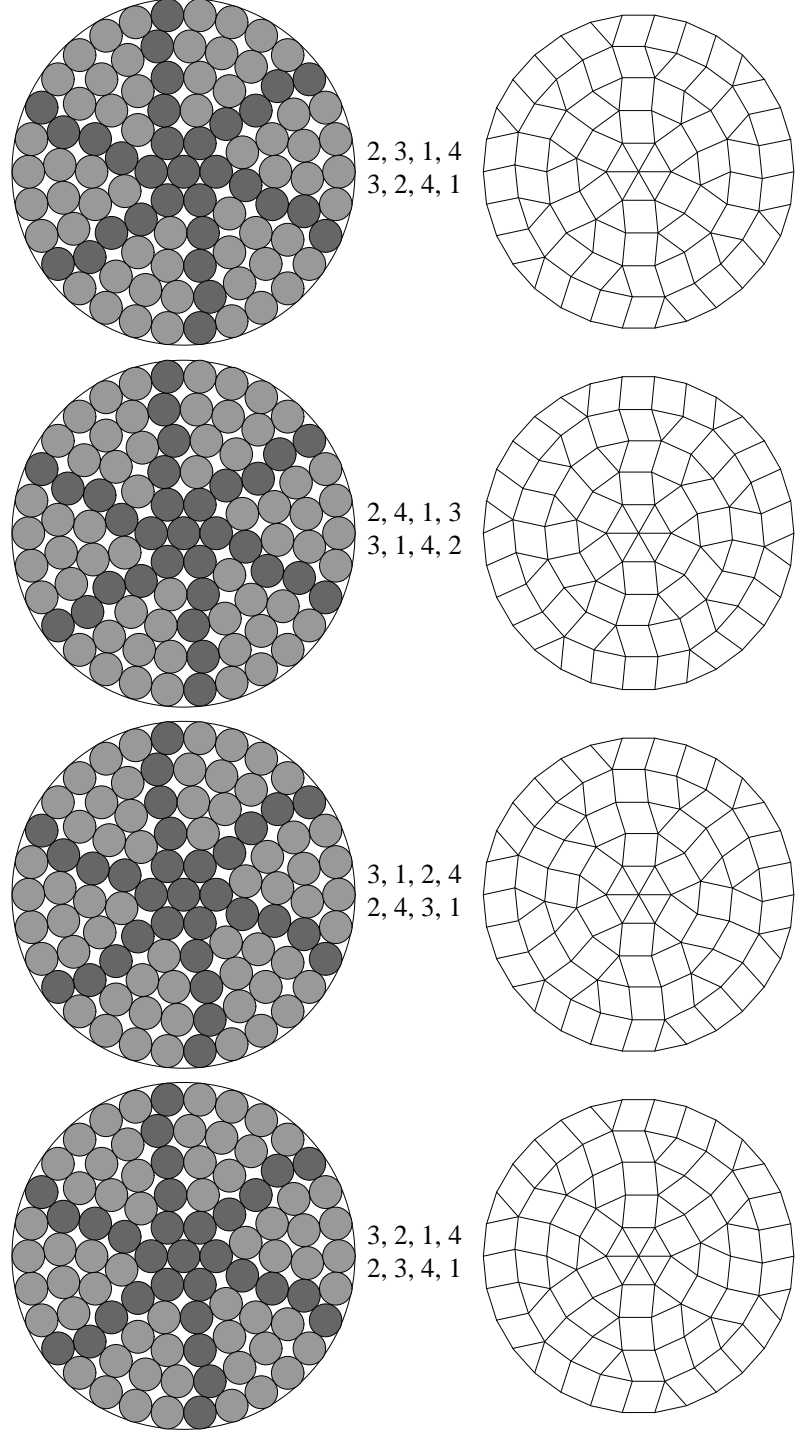

Fig. 3.4. Second four irregular densest (that we found) packings of 91 disks. Each packing shown on the left is accompanied by its two generating sequences in the middle and the bond diagram on the right.

of layer $i$ can only contact two disks in the same layer (the previous and the next disk along the circle) and the disks of layers $i-1$ and $i+1$ (with the central disk being counted as layer 0 and the boundary as layer $k+1$ ).

Note that we distinguish between a configuration and a (rigid) packing. We call a configuration of nonoverlapping disks inside a circle a rigid packing (or simply a packing) if there exists a nonempty subset of disks in the configuration, such that the 
only continuous motion of some or all of disks in the subset is the rotation of this subset as a whole with the center of rotation being at the center of the enclosing circle. Curved hexagonal patterns are rigid packings because each internal $i$ th layer of disks has triangles (as seen on bond diagrams) that connect it to the corresponding outer layer $i+1$, and because the outermost $k$ th layer is obviously rigid.

\section{How the "Billiards" Algorithm Produces Packings}

A detailed description of the philosophy, implementation, and applications of this eventdriven algorithm can be found in [8] and [9]. Essentially, the algorithm simulates a system of $n$ perfectly elastic disks. In the absence of gravitation and friction, the disks move along straight lines, colliding with each other and the region walls according to the standard laws of mechanics, all the time maintaining a condition of no overlap. To form a packing, the disks are uniformly allowed to increase gradually in size, until no significant growth can occur. Figure 4.1 displays four successive snapshots in an experiment with 61 disks. We took the snapshots beginning with the time when a local order begins to form and until the time when the set of neighbors of each disk is stabilized.

The latest snapshot shown in Fig. 4.1, one after 441704 collisions, looks dense and, within the drawing resolution, it is identical (up to a mirror-reflection) to the final snapshot (see Fig. 2.1). However, numerically there are gaps of the order of $10^{-5}$ to $10^{-3}$ of the disk diameter in disk-disk and disk-wall pairs that appear to be in contact with each other in Fig. 4.1. Accordingly, only the first three decimal digits of $D / d$, corresponding to the latest shown in Fig. 4.1, snapshot are identical with the correct $D / d$. To close these gaps and to achieve full convergence of $D / d$ and of the density, it usually takes a 10 to 20 million further collisions. We consider $D / d$ and the density to have converged when their values do not change with full double precision for several million collisions.

Of course, as is typical in numerical iterative convergent procedures, if the computations were performed with infinite precision, the convergence would be never achieved and the ever-diminishing gaps would always be there. The "experimental" converged values agree with the "theoretical" ones computed by formulas (3) and (4) to 14 or more significant digits. Moreover, when we initialize the disk positions differently, then the final parameters achieved are either quite distinct and significantly smaller than those achieved in the run presented in Fig. 4.1-and then the corresponding pattern is different from a curved hexagonal packing - or they are identical to 14 or more significant digits - and then the corresponding final pattern is one of the six known curved hexagonal ones. This makes us suspect that we have found the best possible packing and that its parameters $D / d$ and density are correct to 14 significant digits.

This algorithm does not equally favor the existing curved hexagonal packings. For the chosen algorithm parameter settings, including the slow disk expansion (the ratio of the disk expansion speed to the average linear motion speed is 0.001 ), the overwhelming majority of produced curved hexagonal packings were of the regular patterns. For example, out of our 1002 runs with $n=h(5)=91$ disks, curved hexagonal packings were 


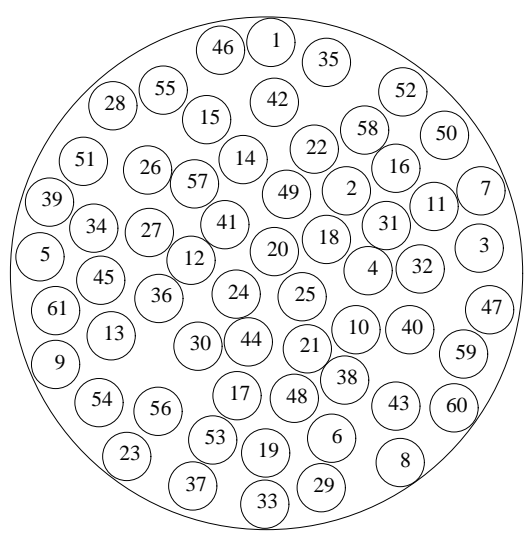

at 16542 collisions

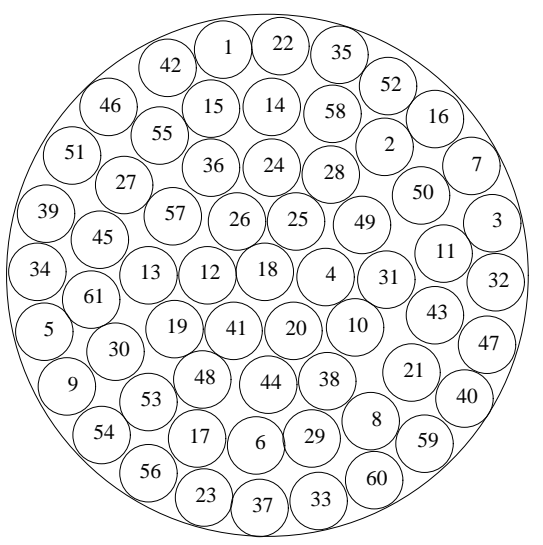

at 53764 collisions

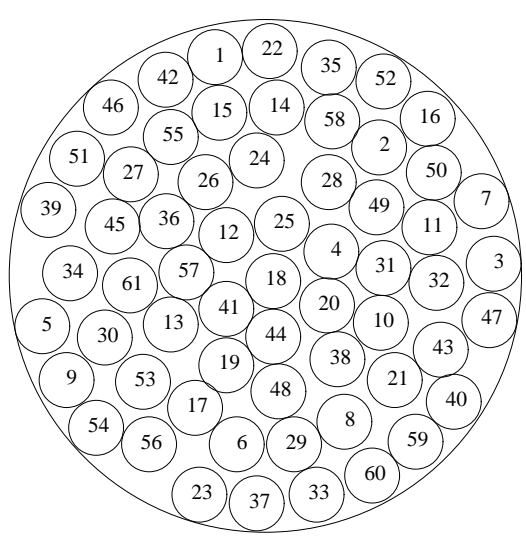

at 36187 collisions

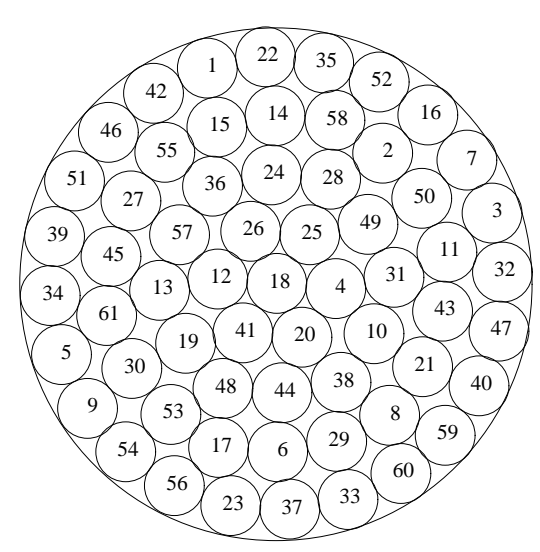

at 441704 collisions

Fig. 4.1. Successive snapshots of the simulating expansion of 61 disks inside a circle. The progress is monitored by counting collisions. Disks are labeled 1 to 61 arbitrarily but the same disk carries the same label in all four snapshots. The last pattern is a mirror-reflection of the packing of 61 disks in Fig. 2.1.

obtained 90 times (9\%). Among those, the four existing regular patterns (33\%) were seen in 81 runs $(90 \%)$, with about the same frequency each. Only three out of the existing eight irregular patterns were seen in the remaining nine runs. We gave up waiting for the other five irregular patterns, shown in Figs. 3.3 and 3.4, to be generated spontaneously, i.e., from random initial configurations. Instead, we constructed those from their path sequences, using the method discussed in Section 3. 


\section{Packings of 127,169 , and 217 Disks}

We ran the "billiards" algorithm for $n=h(6)=127, n=h(7)=169$, and $n=h(8)=$ 217 disks. For these $n$ the algorithm produced better packings than the curved hexagonal ones. The patterns of those packings can all be described as a (possibly disturbed) hexagonal disk assembly in the middle, surrounded with irregularly placed disks at the circular border. For larger $n$ this common pattern becomes more evident. As an example, we show in Fig. 5.1 the best packing we obtained for 127 disks.

We believe the packings achieved for $n=127,169$, and 217 are stable. But we do not think they are the best, because of a large number of local minima for these $n$. For example, the packing shown in Fig. 5.1 was the best among 111 independent tries. Each try resulted in a packing which is distinct from the others, and had distinct parameters

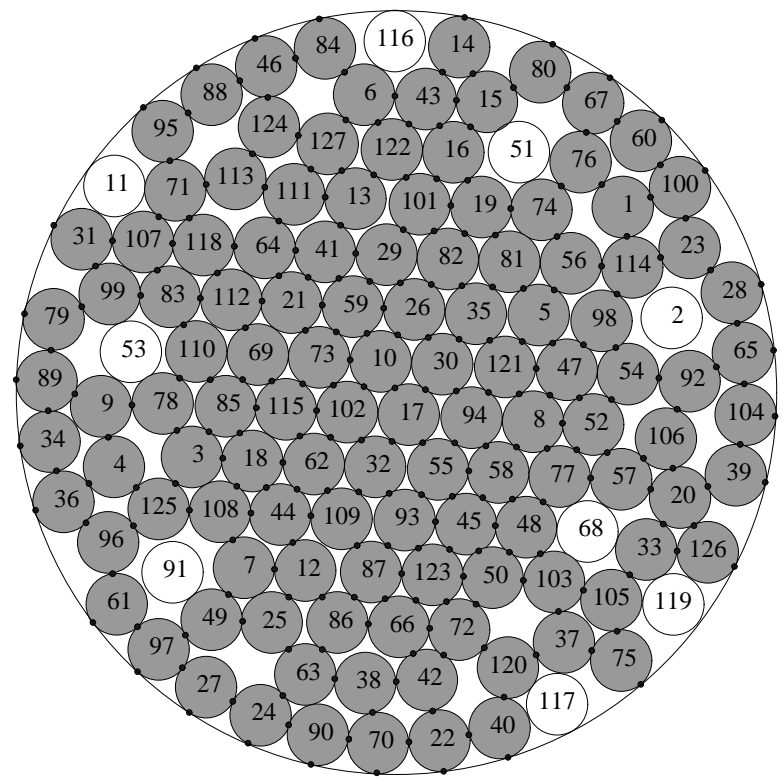

127 disks (244 bonds; 9 rattlers) density $=0.81755666415904$

$D / d=12.463583540213$

Fig. 5.1. A packing of $h(6)=127$ disks in a circle that is better than the corresponding curved hexagonal packings. Each disk is provided with its unique identification label to facilitate the reference. Little black dots are "bonds"; a bond indicates that the corresponding distance is less than $10^{-13}$ of the disk diameter. Where a pair disk-disk or disk-wall are apparently in contact but no bond is shown, e.g., between disks 3 and 108, the computed distance is at least $10^{-5}$ of the disk diameter. The shaded disks cannot move given the positions of their neighbors, the nonshaded are "rattlers" that are free to move within their confines. It might seem that there are sufficiently large cavities near disks 27, 104, 28, and 88, that are in contact with the boundary, so that if the disks are pushed into the cavities the packing will "unjam." This does not happen. For example, pushing disk 27 into the position of contact with 97 and 49 results in the overlap with 24 . 
Table 5.1. Curved hexagonal packings versus the experimental ones for $k=6,7$, and 8 .

\begin{tabular}{lccc}
\hline$k$ & 6 & 7 & 8 \\
$n$ & 127 & 169 & 217 \\
\hline Curved hexagonal density & 0.81622935362082 & 0.81710701192903 & 0.81776562948873 \\
Curved hexagonal $D / d$ & 12.473713245670 & 14.381489999655 & 16.289788298679 \\
\hline Experimental density & 0.81755666415904 & 0.82672262170717 & 0.83499393075147 \\
Experimental $D / d$ & 12.463583540213 & 14.297609837687 & 16.120860041887 \\
Better packings & 14 out of 111 & all 70 & all 62 \\
\hline
\end{tabular}

$D / d$ and density. We found 14 out of 111 packings were better than the curved hexagonal.

These runs for $h(k)$ disks, $k>5$, were in contrast to the runs for $n=h(5)=91$ and smaller $n$. For example, for 91 disks, the best (curved hexagonal) packings were obtained 90 times out of 1002 with parameters $D / d$ and density, agreeing to 15 significant places. The results of packings for $h(k), k>5$, disks are summarized in Table 5.1.

\section{Tightness of Curved Hexagonal Packings}

Figures 6.1 and 6.2 depict the best found packings of $n=h(k)-1$ and $h(k)+1$ disks for $k=2,3,4$, and 5, that is, for $n=18,20,36,38,60,62,90$, and $92 .{ }^{3}$ The known packings of $n=6$ and 8 disks have to the same tendencies, namely:

(a) the pattern of dense packing of $n=h(k)-1$ disks is obtained by removing one disk from the pattern of dense packing of $h(k)$ disks (which is a curved hexagonal packing for the considered $k \leq 5$ ) and, for $k \geq 3$, by a small rearrangement of the disks; its parameter $D / d$ is either not changed (for $k=1$ and 2) or is decreased only slightly (for $k=3,4$, and 5); and

(b) the pattern of dense packing of $n=h(k)+1$ disks differs significantly from the pattern of dense packing of $h(k)$ disks and its parameter $D / d$ is increased substantially for all $k=1,2,3,4$, and 5).

The changes in $D / d$ are "slight" and "substantial" only in comparison to each other. The ratio of the decrease of $D / d$ for $n=h(k)-1$ over the increase of $D / d$ for $n=h(k)+1$ is given in Table 6.1.

\section{Discussion}

Our experiments reveal that for sufficiently large $n$, good packings of $n$ equal disks in a circle have a complex pattern like that in Fig. 5.1, with a large, perhaps disturbed, core of

\footnotetext{
3 The forthcoming paper [6] lists nine more distinct packings of 18 disks that are equal in quality to the one presented in Fig. 6.1.
} 


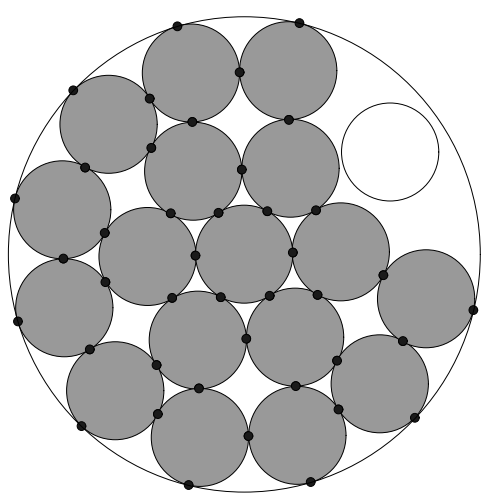

18 disks (41 bonds, 1 rattler) density $=0.76091887384428$ $\mathrm{D} / \mathrm{d}=4.8637033051564$

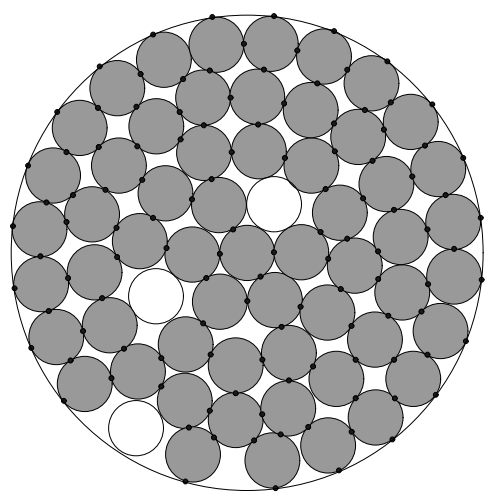

60 disks (115 bonds, 3 rattlers) density $=0.80259916264035$ $\mathrm{D} / \mathrm{d}=8.6462198454579$

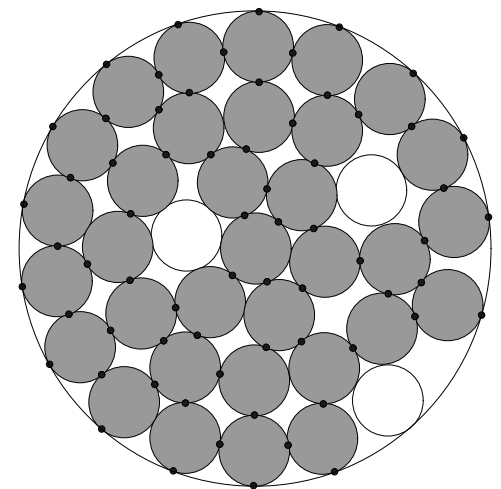

36 disks ( 66 bonds, 3 rattlers) density $=0.79088397691361$ $\mathrm{D} / \mathrm{d}=6.7467537934242$

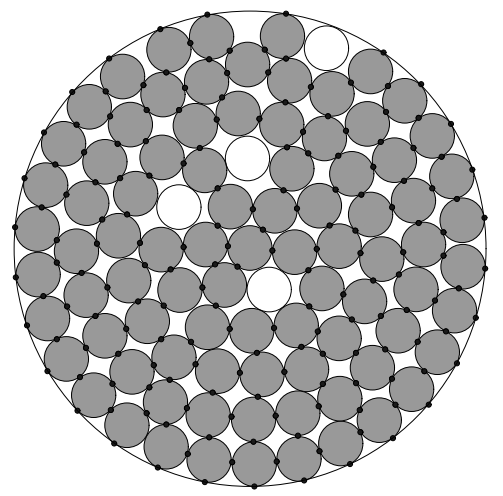

90 disks (172 bonds, 4 rattlers) density $=0.80921006858796$ $\mathrm{D} / \mathrm{d}=10.546069177954$

Fig. 6.1. The best found packings of $n=h(k)-1$ disks for $k=2,3,4$, and 5 ( $n=18,38$, 60, and 90).

hexagonally packed disks and irregularly placed disks along the periphery. The fraction of the irregular peripheral disks and the perturbation in the hexagonally packed core usually diminish with $n$. It is very difficult to obtain the best packings for large $n$, but we would guess their pattern to be of the same irregular type. On the other hand, for $n \leq 25$, symmetric and regular patterns of the best packings have been previously observed that do not obey the general description given above for large $n$. Our computer experiments show that at least for a particular class of $n=h(k)=3 k(k+1)+1$, the transition from the regular, here curved hexagonal, pattern to the irregular core-hexagonal pattern occurs between $n=91$ and $n=127$. 


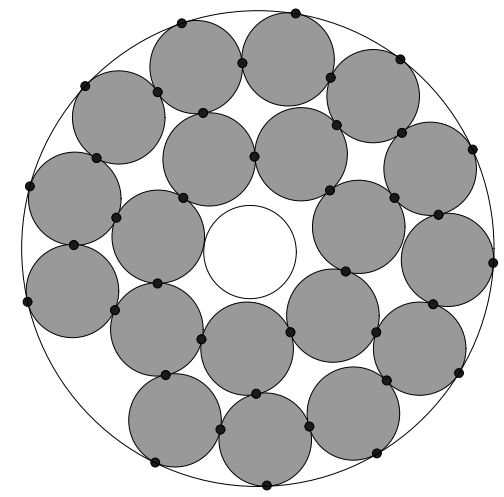

20 disks ( 38 bonds, 1 rattler) density $=0.76224828956498$ $D / d=5.12232073699150$

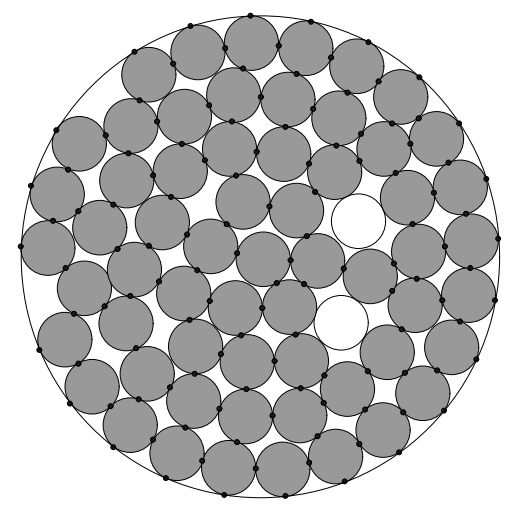

62 disks ( 120 bonds, 2 rattlers) density $=0.79523110498328$ $D / d=8.82976540897204$

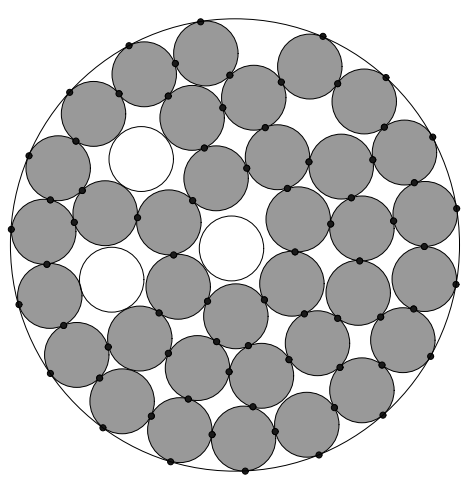

38 disks (70 bonds, 3 rattlers) density $=0.78402454881084$ $D / d=6.96188696522814$

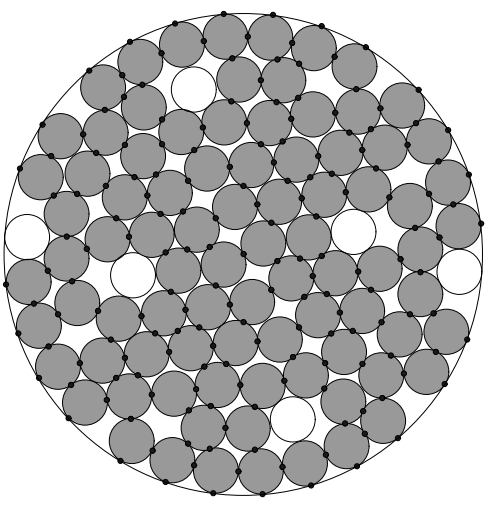

92 disks (172 bonds, 6 rattlers) density $=0.80586815498812$ $D / d=10.68468975902245$

Fig. 6.2. The best found packings of $n=h(k)+1$ disks for $k=2,3,4$, and $5(n=20,38,62$, and 92).

Table 6.1. The ratio of the decrease of $D / d$ for $n=h(k)-1$ over its increase for $n=h(k)+1$.

\begin{tabular}{lrrrrr}
\hline$k$ & 1 & 2 & 3 & 4 & 5 \\
\hline$n=h(k)$ & 7 & 19 & 37 & 61 & 91 \\
\hline Ratio & 0 & 0 & 0.0592 & 0.0895 & 0.1755 \\
\hline
\end{tabular}




\section{References}

1. H. T. Croft, K. J. Falconer, and R. K. Guy, Unsolved Problems in Geometry, Springer-Verlag, Berlin, 1991, pp. 107-111.

2. L. Fejes Tóth, Lagerungen in der Ebene, auf der Kugel und im Raum, 2nd edn., Springer-Verlag, Berlin, 1972.

3. J. H. Folkman and R. L. Graham, A packing inequality for compact convex subsets of the plane, Canadian Mathematical Bulletin 12 (1969), 745-752.

4. M. Goldberg, Packing of 14, 16, 17, and 20 circles in a circle, Mathematical Magazine 44 (1971), 134-139.

5. R. L. Graham and B. D. Lubachevsky, Dense packings of equal disks in an equilateral triangle: From 22 to 34 and beyond, The Electronic Journal of Combinatorics 2 (1995), \#A1.

6. R. L. Graham, B. D. Lubachevsky, K. J. Nurmela, and P. R. J. Östergård, Dense packings of congruent circles in a circle, Discrete Mathematics (to appear).

7. S. Kravitz, Packing cylinders into cylindrical containers, Mathematical Magazine 40 (1967), 65-71.

8. B. D. Lubachevsky, How to simulate billiards and similar systems, Journal of Computational Physics 94 (1991), 255-283.

9. B. D. Lubachevsky and F. H. Stillinger, Geometric properties of random disk packings, Journal of Statistical Physics 60 (1990), 561-583.

10. N. Oler, A finite packing problem, Canadian Mathematical Bulletin 4 (1961), 153-155.

11. G. E. Reis, Dense packing of equal circles within a circle, Mathematical Magazine 48 (1975), 33-37.

Received May 15, 1995, and in revised form March 5, 1996. 